\title{
BMJ Open Effect of the 2016 Kumamoto earthquakes on preventable hospital admissions: a retrospective cohort study in Japan
}

\author{
Yusuke Sasabuchi, ${ }^{1}$ Hiroki Matsui, ${ }^{1,2}$ Kazuhiko Kotani, ${ }^{3}$ Alan Kawarai Lefor, ${ }^{4}$ \\ Hideo Yasunaga ${ }^{1,2}$
}

To cite: Sasabuchi Y, Matsui $\mathrm{H}$, Kotani $\mathrm{K}$, et al. Effect of the 2016 Kumamoto earthquakes on preventable hospital admissions: a retrospective cohort study in Japan. BMJ Open 2018;8:e021294. doi:10.1136/ bmjopen-2017-021294

- Prepublication history and additional material for this paper are available online. To view these files, please visit the journal online (http://dx.doi. org/10.1136/bmjopen-2017021294).

Received 29 December 2017 Revised 19 May 2018 Accepted 15 June 2018

A Check for updates

(C) Author(s) (or their employer(s)) 2018. Re-use permitted under CC BY-NC. No commercial re-use. See rights and permissions. Published by BMJ.

${ }^{1}$ Data Science Center, Jichi Medical University, Tochigi, Japan

${ }^{2}$ Department of Clinical Epidemiology and Health Economics, Graduate School of Medicine, The University of Tokyo, Tokyo, Japan ${ }^{3}$ Division of Community and Family Medicine, Jichi Medical University, Tochigi, Japan

${ }^{4}$ Department of Surgery, Jichi Medical University, Tochigi, Japan

Correspondence to Dr Yusuke Sasabuchi; sasabuchi-tky@umin.ac.jp

\section{ABSTRACT}

Background and objectives The Kumamoto earthquakes struck Kumamoto prefecture, in the southwest part of Japan in April 2016. Physical and mental disorders presenting to hospital increased after the 2016 Kumamoto earthquakes. Impaired access to primary care due to the earthquakes may have contributed to this increase. However, it is not known whether the 2016 Kumamoto earthquakes affected access to primary care. The objective of the present study was to investigate the impact of the 2016 Kumamoto earthquakes on short-term health conditions by analysing ambulatory care sensitive conditions (ACSCs), using administrative data from Kumamoto prefecture.

Design A retrospective cohort study.

Setting Residents enrolled in National Health Insurance or Late Elders' Health Insurance from Kumamoto prefecture, Japan.

Participants All hospital admissions due to ACSCs between 15 March and 16 May in each year from 2013 to 2016.

Outcome measures ACSCs are defined as conditions for which appropriate primary care interventions could prevent admission to the hospital.

Results We identified a total of 7921, 18763 and 85436 admissions for vaccine, acute and chronic preventable ACSCs, respectively, during the study period. Admissions within 7 days after the 2016 Kumamoto earthquakes increased to $32.6 \%(10.2,59.5), 44.1 \%(27.0,63.5)$ and $27.7 \%(20.2,35.6)$ for vaccine-preventable, acute and chronic ACSCs, respectively. However, admissions for ACSCs did not change significantly 30 days after the earthquakes.

Conclusion The 2016 Kumamoto earthquakes were associated with increased hospital admissions for ACSCs. The impact of the earthquakes on admissions for ACSCs did not persist for more than 7 days.

\section{INTRODUCTION}

The 2016 Kumamoto earthquakes struck Kumamoto prefecture, in the southwest part of Japan. Two earthquakes, of Japanese seismic intensity scale 7, occurred in April 2016. The earthquake sequence consisted

\section{Strengths and limitations of this study}

- This study is first to evaluate the effects of the 2016 Kumamoto earthquakes on preventable hospital admissions.

- The database does not include information related to the degree of damage by the earthquakes in different areas within Kumamoto prefecture.

- The data for patients who enrolled in other health insurance systems were not included in the database.

- The use of these ambulatory care sensitive conditions in Japanese primary care settings remains to be further studied for validity.

of a powerful foreshock on 14 April (magnitude 6.5) followed by a larger mainshock on 16 April (magnitude 7.3). A total of 183882 residents were evacuated to 855 shelters (the majority of the shelters were elementary or junior high schools) just after the earthquakes and 1 week later, the number of evacuees was approximately $70000 .{ }^{1}$ As of 13 April 2017, there were 228 fatalities and approximately 200000 homes were completely destroyed, half-destroyed or partially destroyed. ${ }^{1}$ Many hospitals and clinics were also partially destroyed and lost their capacity to function. Of 2530 medical and dental clinics, structures or equipment were destroyed in 1302 institutions. $^{2}$

Ambulatory care sensitive conditions (ACSCs) are defined as conditions for which appropriate intervention in primary care could obviate the need for hospital admission. ${ }^{34}$ ACSCs have been used as indicators of the accessibility and quality of primary care. ${ }^{5}$ Previous studies have reported that physical ${ }^{6-8}$ and mental ${ }^{910}$ disorders presenting to hospital increased after the earthquakes. Similarly, increased incidence of stroke, ${ }^{11}$ cardiovascular disease, ${ }^{12}$ pneumonia, ${ }^{12}$ exacerbation of Parkinson's disease ${ }^{13}$ and seizures ${ }^{14}$ has 
been reported after the 2016 Kumamoto earthquakes. Impaired access to primary care due to the earthquakes may have contributed to this increase. On the basis of the data on the number of destroyed clinics, access to primary care probably decreased after the earthquakes. However, the impact of the 2016 Kumamoto earthquakes on access to primary care has not been studied.

The objective of the present study was to investigate the impact of the 2016 Kumamoto earthquakes on short-term health conditions with regard to ACSCs, using an administrative database from Kumamoto prefecture.

\section{METHODS}

\section{Data source and population}

In this retrospective cohort study, patient data were extracted from two claims databases. The first database belongs to the National Health Insurance of Kumamoto prefecture, which is a community-based health insurance plan, and mainly insures self-employed, the irregularly employed, pensioners younger than 75 years and their families. The second database is from the Late Elders' Health Insurance, which is a health insurance plan for people aged 75 years or older. These plans include approximately 800000 enrolees, representing about $50 \%$ of the residents of Kumamoto prefecture. According to the census, the population in Kumamoto prefecture before the earthquakes was 1813000 , and 6 months after the earthquakes it decreased by approximately 6000 . The enrolees are older and have lower incomes than the average population in Japan. The database includes patient-level demographic data, a date-stamped diagnosis coded according to the International Classification of Diseases 10th Revision (ICD-10) and date-stamped inpatient and outpatient medical and pharmacy claims (procedure and prescription codes). All data were anonymised and deidentified after generating a pseudonymised number specific to the database.

We extracted data for patients from all hospital admissions due to ACSCs between 15 March and 16 May of each year from 2013 to 2016, which includes the dates for 30 days before the foreshock to 30 days after the mainshock. We defined the period between 15 March 2016 and 13 April 2016 as 'before the quake' and the period from 17 April 2016 to 16 May 2016 as 'after the quake'. We defined the corresponding data of the years from 2013 to 2015 as the control period. Since the impact of the earthquakes on ACSCs was estimated to decrease with time, we also estimated the impact of the earthquakes on ACSCs for 1 week before and after the earthquakes.

\section{Ambulatory care sensitive conditions}

ACSCs are defined as conditions for which an appropriate primary care intervention could prevent admission to the hospital. ${ }^{34}$ ACSCs have been used as indicators of the accessibility and quality of primary care. ${ }^{5}$ High frequencies of admissions for ACSCs indicate poor coordination between primary and secondary care. An avoidable admission for an ACSC indicates poor overall quality of care, even if the ACSC episode itself is well managed. ${ }^{4}$ The outcomes of this study compared before the quake and after the quake hospital admissions for the following three types of ACSCs: (1) vaccine-preventable ACSCs (where immunisation and other interventions can prevent illness, eg, influenza and pneumonia); (2) chronic ACSCs (where effective care can prevent flare-ups, eg, asthma, congestive heart failure, diabetes complications, chronic obstructive pulmonary disease, angina, iron deficiency anaemia, hypertension and nutritional deficiency) and (3) acute ACSCs (where early intervention can prevent more serious progression, eg, dehydration and gastroenteritis; pyelonephritis; perforated/bleeding ulcer; cellulitis; pelvic inflammatory diseases; ear, nose and throat infections; dental conditions; convulsions and epilepsy; and gangrene). Online supplementary table 1 shows the ICD-10 codes used to identify the 19 ACSCs commonly used in the National Health Service in the $\mathrm{UK}^{3}$

\section{Statistical analysis}

Continuous variables are presented as the median and IQR. A locally weighted scatterplot smoothing curve was drawn with the daily number of admissions for ACSCs. The associations between the earthquake and subsequent ACSCs are evaluated using a difference-in-differences design by using a comparison group that is experiencing the same trends, but was not exposed to the earthquake. In the difference-in-differences design, two differences should be estimated: the difference between before and after 30 days of the earthquake in the year of the earthquake (2016) (D1) and the difference between before and after the date of the earthquake in the years before the earthquake (2013-2015) (D2). The change in outcomes related to the earthquake beyond the background trends can then be estimated using the difference-in-differences analysis as D1-D2. ${ }^{15}$ To estimate the impact of the earthquake on ACSCs, we applied generalised additive models, which are flexible methods for modelling non-linear association, ${ }^{16}$ linked to Poisson regression because the number of daily admissions for ACSCs is counted. Independent variables were periods (before and after the earthquakes), year (the affected year (2016) and before the affected year (2013-2015)) and the interaction of these two variables (to estimate the difference in differences of the number of admissions) in addition to days before or after the earthquake. Previous studies showed that the variety of diseases increased after the Great East Japan Earthquake and Tsunami disaster and decreased within a few weeks. ${ }^{617-20}$ Damage due to the 2016 Kumamoto earthquakes was less extensive and restoration from the damage was more prompt than the Great East Japan Earthquake and Tsunami disaster. Therefore, we also compared changes in ACSC rates 7 days before and after the earthquake. All statistical analysis was conducted using R V.3.4.1 (The R Foundation, Vienna, Austria) and package 'mgcv' was used for generalised additive models. 
Table 1 Number of admissions for ambulatory care sensitive conditions before and after the earthquakes in 2016 and during the corresponding intervals in 2013-2015

\begin{tabular}{lll}
\hline & $3 / 15-4 / 13$ & $4 / 17-5 / 16$ \\
\hline Vaccine preventable & & \\
2013 & $32(20-42)$ & $33(20-40)$ \\
2014 & $35(19-46)$ & $28(16-35)$ \\
2015 & $36(22-42)$ & $28(15-43)$ \\
2016 & $50(25-55)$ & $37(19-54)$ \\
\hline Acute preventable & & \\
\hline 2013 & $85(53-100)$ & $89(41-108)$ \\
\hline 2014 & $88(44-98)$ & $82(38-103)$ \\
2015 & $84(56-101)$ & $81(48-108)$ \\
\hline 2016 & $88(46-109)$ & $88(48-116)$ \\
\hline Chronic preventable & & \\
\hline 2013 & $385(181-468)$ & $406(148-499)$ \\
\hline 2014 & $418(183-471)$ & $409(143-471)$ \\
\hline 2015 & $428(207-508)$ & $399(162-501)$ \\
\hline 2016 & $424(193-471)$ & $403(162-505)$ \\
\hline
\end{tabular}

Number of admissions are presented as median and IQR.

\section{PATIENT AND PUBLIC INVOLVEMENT}

No patients were involved in setting the research question or the outcome measures, nor were they involved in developing plans for design or implementation of the study. We have no plans to involve patients in dissemination.

\section{RESULTS}

A total of 7921, 18763 and 85436 admissions for vaccine, acute and chronic preventable ACSCs, respectively, were reviewed. Table 1 shows the median number and IQR of daily admissions for ACSCs before and after the earthquake in 2016 and the corresponding dates in the previous 3 years.

Figure 1 shows the median daily number of admissions for ACSCs and the IQR before and after the earthquake in 2016 and the corresponding dates in the previous 3 years. In the first 7 days after the earthquakes, all ACSCs in 2016 increased and then decreased, while the number of admissions for ACSCs did not change in the years 2013-2015.

Table 2 shows the results of the difference-in-differences analyses. Per cent changes in difference-in-differences estimates for three ACSCs for 30 days before and after the earthquake were not significant. However, difference-in-differences analysis within 7 days of the earthquakes showed that admissions increased to $32.6 \%$ (10.2, $59.5), 44.1 \%(27.0,63.5)$ and $27.7 \%(20.2,35.6)$ for vaccine-preventable, acute and chronic ACSCs, respectively, after the 2016 Kumamoto earthquakes.

\section{DISCUSSION}

Hospitalisation for ACSCs has been used as an indicator of access to outpatient care. ${ }^{5}$ Using the health insurance records of Kumamoto prefecture, we found that admissions for ACSCs increased within 7 days after the 2016 Kumamoto earthquakes.

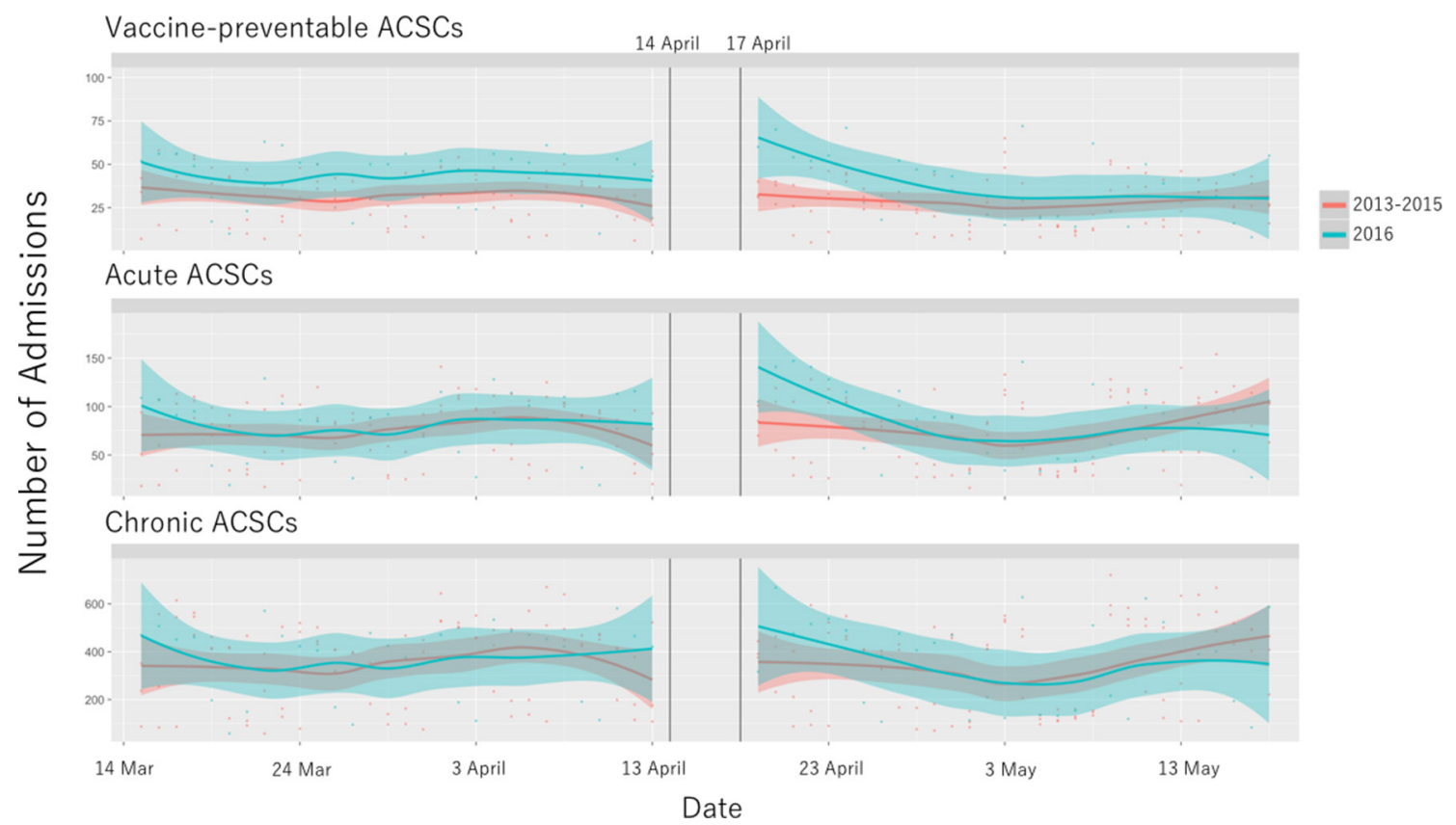

Figure 1 Daily admissions for ambulatory care sensitive conditions (ACSCs) before and after the earthquake and corresponding intervals during the previous 3years (2013-2015). Dots indicate the number of admissions for ACSCs on each day. The blue line indicates locally weighted scatterplot smoothing (LOWESS) curves of the number of admissions for ACSCs in 2016 (the year the earthquakes occurred). The red line indicates LOWESS curves of the number of admissions for ACSCs in the previous 3 years (2013-2015). The grey belts show 95\% Cls. The two black vertical lines indicate the days of the foreshock and mainshock. All ACSCs in 2016 increased during the first several days after the earthquakes and then decreased, while there were no changes in the years 2013-2015. 


\begin{tabular}{|llc|}
\hline Table 2 & Results of difference-in-differences analyses \\
\hline & $\%$ difference $(95 \%$ CI) & P values \\
\hline $\begin{array}{l}\text { 30 days } \\
\text { Vaccine preventable }\end{array}$ & $0.0(-9.2$ to 10.0$)$ & 0.995 \\
Acute preventable & $2.7(-3.7$ to 9.7$)$ & 0.416 \\
\hline $\begin{array}{l}\text { Chronic preventable } \\
\text { days }\end{array}$ & $-0.7(-3.7$ to 2.4$)$ & 0.647 \\
Vaccine preventable & $32.6(10.2$ to 59.5$)$ & 0.003 \\
Acute preventable & $44.1(27.0$ to 63.5$)$ & $<0.01$ \\
Chronic preventable & $27.7(20.2$ to 35.6$)$ & $<0.01$ \\
\hline
\end{tabular}

These results show that admissions for ACSCs increased 7 days after the earthquake, but did not change 30 days after the earthquake. The number of admissions increased just after the disaster, and then immediately decreased thereafter. Impaired access to primary care after the earthquake may have recovered promptly. Previous studies reported that the variety of diseases temporarily increased and started to decrease several weeks after the Great East Japan Earthquake and Tsunami disaster. ${ }^{6-8} 17$ The 2016 Kumamoto earthquakes were different from the Great East Japan Earthquake in that the Kumamoto earthquakes were not accompanied by a tsunami. In addition, the number of residents evacuated to shelters rapidly decreased from 180000 to 70000 within 1 week after the earthquakes. ${ }^{1}$ The area struck by the earthquake seemed to be less severely destroyed and access to primary care may have recovered promptly.

The present study shows that hospitalisations for the three types of ACSCs increased within 7 days after the 2016 Kumamoto earthquakes. The number of patients with vaccine-preventable ACSCs may have increased because shelters were packed with evacuees. A previous study showed that the incidence of shelter-acquired pneumonia increases after an earthquake. ${ }^{21}$ Another study reported that too many people were evacuated to the shelters in Kumamoto prefecture and the cramped accommodations left little room to walk between beds. ${ }^{22}$ Some of the evacuees in these shelters tried not to move into the shelters. ${ }^{22}$ The density of evacuees in shelters may have led to outbreaks of vaccine-preventable ACSCs. Early intervention can prevent progression of acute ACSCs to more serious conditions. It is plausible that evacuees in shelters may have not been able to or may have hesitated to consult doctors about minor ailments. These minor health problems in evacuees may have progressed to more serious conditions resulting in the need for admission to the hospital. Similarly, temporary interruption of the treatment of chronic conditions such as hypertension or diabetes mellitus may cause sudden changes in blood pressure, or serum glucose concentration, ${ }^{23}{ }^{24}$ resulting in the need for hospitalisation for patients with chronic ACSCs. Evacuees often left their medications at home, and clinics in the affected areas lacked medicines. Although Disaster Medical Assistance Teams (DMATs) were dispatched to
Kumamoto prefecture just after the disaster, the purpose of the DMATs was to rescue people who had acute injuries in the first 48 hours. Other medical teams took over the care of patients with subacute to chronic conditions thereafter. Therefore, the care of people with chronic conditions may have been interrupted in the initial phase of the disaster.

These results have important implications for preventing avoidable admissions in postdisaster settings. Focusing on the early period after disaster may be important for the health of residents who live in the affected area. For example, avoiding overcrowding in shelters, creating a place where physicians can see patients and providing continuity of care for chronic conditions may prevent avoidable admissions in the early phase after a disaster. Further research is warranted to develop approaches to improve access to primary care and reduce avoidable hospital admissions after an earthquake or other natural disasters.

We acknowledge that the present study has several limitations. First, the database does not include information related to the degree of damage by the earthquakes in different areas within Kumamoto prefecture. Second, the data for patients who enrolled in other health insurance systems were not included in the database. Estimating the number of admissions for ACSCs in the entire area using age and gender standardisation may not be accurate because of differences between the population included in the present study and the general population. Third, the use of these ACSCs, derived from data in the UK, in Japanese primary care settings remains to be further studied for validity. Fourth, hospitals may have changed their admission criteria for people with ACSCs in the population included in this study because doctors hesitated to send older patients back to the shelters where the level of care was much lower than normal. Fifth, ACSCs are not a direct measure of the impaired of access to primary care. A consensus has not been reached whether ACSCs are appropriate indicators for access to primary care. ${ }^{25} 26$

In conclusion, the 2016 Kumamoto earthquakes were associated with an increased number of hospital admissions for ACSCs. The impact of the earthquakes on admissions for ACSCs did not persist for more than 7 days.

Contributors YS and HY were involved in conception, study design, data collection, analysis, interpretation of the results and drafting the manuscript. HM was involved in conception, study design, data collection, data cleaning, analysis and interpretation of the manuscript. KK was involved in conception, data collection and interpretation of the results. AKL was involved in conception, interpretation of the results and drafting the manuscript. YS, HY, HM and KK designed the protocol and collected data. HY, HM, AKL and KK revised the first draft of the manuscript. All authors interpreted the results and approved the final version of the manuscript prior to its submission.

Funding This work was funded by ImPACT Program of Council for Science, Technology and Innovation (Cabinet Office, Government of Japan).

Competing interests None declared.

Patient consent Not required.

Ethics approval The Institutional Review Board of Jichi Medical University. 
Provenance and peer review Not commissioned; externally peer reviewed.

Data sharing statement The datasets generated during and/or analysed during the current study are only available for researchers who participated and registered in the ImPACT Program.

Open access This is an open access article distributed in accordance with the Creative Commons Attribution Non Commercial (CC BY-NC 4.0) license, which permits others to distribute, remix, adapt, build upon this work non-commercially, and license their derivative works on different terms, provided the original work is properly cited, appropriate credit is given, any changes made indicated, and the use is non-commercial. See: http://creativecommons.org/licenses/by-nc/4.0/.

\section{REFERENCES}

1. Cabinet Office Government of Japan. White Papar on Disaster Management in Japan. 2018;2017 http://www.bousai.go.jp/kyoiku/ panf/pdf/WP2017_DM_Full_Version.pdf

2. Kumamoto Prefectural Government. Damage from the Kumamoto earthquakes. https://www.pref.kumamoto.jp/common/ UploadFileOutput.ashx?c_id=3\&id=19236\&sub_id=1\&flid=100871 (accessed 22 Mar 2018).

3. Purdy S, Griffin T, Salisbury C, et al. Prioritizing ambulatory care sensitive hospital admissions in England for research and intervention: a Delphi exercise. Prim Health Care Res Dev 2010;11:41-50.

4. Kings Fund Data Briefing: Emergency Hospital Admissions for Ambulatory Care Sensitive Conditions. https://www.kingsfund. org.uk/sites/default/files/field/field_publication_file/data-briefingemergency-hospital-admissions-for-ambulatory-care-sensitiveconditions-apr-2012.pdf (accessed 22 Mar 2018).

5. Laditka JN, Laditka SB. Race, ethnicity and hospitalization for six chronic ambulatory care sensitive conditions in the USA. Ethn Health 2006;11:247-63.

6. Aoki T, Fukumoto Y, Yasuda S, et al. The Great East Japan Earthquake Disaster and cardiovascular diseases. Eur Heart $J$ 2012;33:2796-803.

7. Itabashi R, Furui E, Sato S, et al. Incidence of cardioembolic stroke including paradoxical brain embolism in patients with acute ischemic stroke before and after the Great East Japan Earthquake. Cerebrovasc Dis 2014;37:431-7.

8. Yamanda S, Hanagama M, Kobayashi S, et al. The impact of the 2011 Great East Japan Earthquake on hospitalisation for respiratory disease in a rapidly aging society: a retrospective descriptive and cross-sectional study at the disaster base hospital in Ishinomaki. BMJ Open 2013;3:e000865.

9. Yokoyama Y, Otsuka K, Kawakami N, et al. Mental health and related factors after the Great East Japan earthquake and tsunami. PLoS One 2014;9:e102497.

10. Kwon YS, Maruyama S, Morimoto K. Life events and posttraumatic stress in Hanshin-Awaji earthquake victims. Environ Health Prev Med 2001;6:97-103.
11. Inatomi $Y$, Nakajima M, Yonehara $T$, et al. Clinical characteristics of patients with ischemic stroke following the 2016 Kumamoto earthquake. J Clin Neurosci 2017;46:79-84.

12. Yasuda O, Ikeda Y, Ohishi M. Kumamoto Earthquake and health issues of the elderly. Nihon Ronen Igakkai Zasshi 2017;54:120-4.

13. Hori $\mathrm{H}$, Kuratomi $\mathrm{A}$, Ishizaki $\mathrm{M}$, et al. The effects of the Kumamoto earthquake on the clinical symptoms of patients with Parkinson's disease. Rinsho Shinkeigaku 2017;57:425-9.

14. Inatomi $\mathrm{Y}$, Nakajima $\mathrm{M}$, Yonehara $\mathrm{T}$, et al. Clinical characteristics of patients seizure following the 2016 Kumamoto earthquake. J Clin Neurosci 2017;40:123-9.

15. Dimick JB, Ryan AM. Methods for evaluating changes in health care policy: the difference-in-differences approach. JAMA 2014;312:2401-2.

16. Hastie T, Tibshirani R. Generalized additive models for medical research. Stat Methods Med Res 1995;4:187-96.

17. Aoki T, Takahashi J, Fukumoto Y, et al. Effect of the Great East Japan Earthquake on cardiovascular diseases--report from the 10 hospitals in the disaster area. Circ J 2013;77:490-3.

18. Aoyagi T, Yamada M, Kunishima H, et al. Hatta M1, Endo S, Arai K, Inomata S, Gu Y, Kanamori H, Kitagawa M, Hirakata Y, Kaku M. Characteristics of infectious diseases in hospitalized patients during the early phase after the 2011 great East Japan earthquake: pneumonia as a significant reason for hospital care. Chest 2013;143:349-56.

19. Kobayashi S, Hanagama M, Yamanda S, et al. Impact of a largescale natural disaster on patients with chronic obstructive pulmonary disease: the aftermath of the 2011 Great East Japan Earthquake. Respir Investig 2013;51:17-23.

20. Kanno T, lijima K, Abe Y, et al. Peptic ulcers after the Great East Japan earthquake and tsunami: possible existence of psychosocial stress ulcers in humans. J Gastroenterol 2013;48:483-90.

21. Suzuki M, Uwano C, Ohrui T, et al. Shelter-acquired pneumonia after a catastrophic earthquake in Japan. J Am Geriatr Soc 2011;59:1968-70.

22. Maeda K, Shamoto H, Furuya S. Feeding support team for frail, disabled, or elderly people during the early phase of a disaster. Tohoku J Exp Med 2017;242:259-61.

23. Kirizuka K, Nishizaki H, Kohriyama K, et al. Influences of The Great Hanshin-Awaji Earthquake on glycemic control in diabetic patients. Diabetes Res Clin Pract 1997;36:193-6.

24. Greenough PG, Lappi MD, Hsu EB, et al. Burden of disease and health status among Hurricane Katrina-displaced persons in shelters: a population-based cluster sample. Ann Emerg Med 2008;51:426-32.

25. Rosano A, Loha CA, Falvo R, et al. The relationship between avoidable hospitalization and accessibility to primary care: a systematic review. Eur J Public Health 2013;23:356-60.

26. Vuik SI, Fontana G, Mayer E, et al. Do hospitalisations for ambulatory care sensitive conditions reflect low access to primary care? An observational cohort study of primary care usage prior to hospitalisation. BMJ Open 2017;7:e015704. 